\title{
NOÇÕES DO PROCEDIMENTO MONITÓRIO BRASILEIRO PARA OS ITALIANOS
}

\section{SLIGHT KNOWLEDGE OF THE MONITÓRIO PROCEDURE FOR ITALIANS}

\author{
Olavo de Oliveira Neto"
}

\begin{abstract}
Resumo: Trata-se de estudo de direito comparado, representado por aula ministrada na Universidade de Estudos de Milão - Itália, traçando uma análise comparativa entre a ação monitória brasileira e o procedimento de injunção italiano. São abordados os aspectos semelhantes e divergentes de ambos sistemas legais, com breve análise daquilo que a doutrina entende no tocante a aplicação dos preceitos.
\end{abstract}

Palavras-Chave: Direito Comparado: Brasil/Itália. Ação Moritória.

\begin{abstract}
This is a study about comparative jurisprudence, represented for lesson taught in the University of Studies of Milan - Italy, tracing a comparative analysis between the Brazilian "monitória" action and the Italian procedure of injunction. The similar and divergent aspects of both legal systems are boarded, with brief analysis of that the doctrine understands in regards to application of the rules.
\end{abstract}

Key-words: Comparative jurisprudence: Brazil/Italy, "Moritória" action.

\footnotetext{
"Mestre e Doutor pela PUCSP. Pós-doutorado pela Università degli Studi di Milano. Professor de Direito Processual Civil dos cursos de graduação, especialização e mestrado da ITE de Bauru, do COGEAI- PUC/SP, de vários cursos de especialização e da Escola da Magistratura do Paraná. Vencedor do Prêmio "Professor Nota 10" de
} 


\section{1 À GUISA DE ESCLARECIMENTO}

Há cinco anos, a convite do Prof. Avv. Edoardo Flavio Ricci, titular de uma das três cátedras de processo civil da Facoltà degli Studi di Milano, embarcava eu para a Itália, com a finalidade de realizar estudos comparativos entre o direito processual civil italiano e o direito processual civil brasileiro.

Durante quase um ano de convívio, estudando o processo civil italiano e frequentando as aulas de Edoardo Ricci, Giuseppe Tarzia, Achile Saletti e Elena Merlin, todos professores internacionalmente conhecidos, várias foram as lições de vida e da estrutura do sistema que tive o privilégio de conhecer, o que colaborou sobremaneira para minha formação acadêmica.

Recentemente, em conversa com membros do CECIPRO (Centro de estudos de Direito Civil e Processual Civil), revelei que durante a realização de meus estudos tive a oportunidade de proferir algumas palestras na Itália, comparando o nosso sistema legal ao sistema legal italiano; ocasião em que nasceu a idéia de publicar as anotações sobre as aulas ministradas, trazendo à público seu conteúdo, com a finalidade de obtenção de uma visão dos institutos naquele País.

Logo após tais fatos, fomos todos supreendidos com o falecimento do profesor Giuseppe Tarzia, no primeiro semestre deste ano, o que deixou vago entre os processualistas um espaço não preenchível por outrem, na medida em que o saudoso professor era considerado uma das figuras mais brilhantes de sua época.

Transcrevo em seguida, pois, para por em prática a idéia nascida entre os colegas e em como uma pequena e suingela homenagem ao professor Giuseppe Tarzia, a aula proferida a pedido do Prof. Avv. Achile Saletti, em 26 de novembro de 1999, no Corso di diritto processuale civile avanzato da Facultà degli Studi di Milano, quando foi realizada uma análise comparativa entre a nossa ação monitória e o procedimento di ingiunzione italiano.

Também em homenagem aos demais professores citados, para com quem o sentimento de saudade hoje quase se equipara ao sentimento de admiração, transcrevo o texto base na íntegra e em italiano, assim como foi distribuido aos alunos, como demonstração de agradecimento pelo fecundo convívio. 


\section{NOZIONI SULL PROCEDIMENTO D'INGIUNZIONE BRASILIANO}

Buon giorno. Mi piacerebbe, prima di tutto, ringraziare il professore Achile Saletti per l'invito per parlare com tutti voi sull' procedimento d'ingiunzione brasiliano, che là si chiama ação monitória.

Però, prima di parlare sul proprio argomento, credo che sai necessario parlare un pò sull il diritto processuale civile brasiliano, che nella sua strutura ha molta identità com il diritto processuale civile italiano.

Il dirito brasiliano discèndere del diritto portoghese, che del suo stesso posto ha cercato inspirazione nel sistema romano-tedesco, nato nell 1064 nella Università di Bologna.

Ma non basta. Abbiamo avuto il privilegio di tenere con noi, durante qualchè tempo, il professore Enrico Tulio Libman, che ha datto lezioni nella Facoltà di Diritto della Università di San Paolo.

In verità, la permanenza del professore in Brasile ha fatto nascere un gruppo di giuristi oggi conosciuto in tutto il mondo, che ha ricevuto la denominazione di "Scuola processuale di San Paolo".

Nel suo risguardo (rispeto) ha detto il professore Frederico Marques (1977, p. 75) nell' opera intitolata $O$ direito processual civil em São Paulo:

Foi ele o 'mestre' da escola processual de São Paulo, e, como tal, o seu grande guia e orientador. É, por isso, que não se pode falar nessa escola e no desenvolvimento dos estudos processuais em São Paulo, na atualidade, sem mencionar, com letras de ouro, o nome ilustre dessa grande figura que tanto tem enriquecido a literatura jurídica, no campo do Direito Processual Civil.

\section{Ossia:}

È stato il 'maestro'della scuola processuale di San Paolo e così il suo grande guida ed orientadore. È per questo che non si può parlare in questa scuola opure nel svilupo degli studi processuali in San Paolo, nell' attualità, senza menzionare, com letterà di oro, il nome illustre di questa grande persona che tanto ha arricchuto la letteratura giuridica nel campo del diritto processuale civile. 
Occorre che, uno degli aluni di Liebman in quest' epoca, il professore Alfredo Buzaid, dopo si è diventato Ministro della Giustizia e allora è stato incaricato di fare il progetto del nuovo codice di procedura civile brasiliano. Questo progetto si è diventato il codice del 1973. Niente più naturale, così, che parte degli opinioni di Liebman fosseno ricevute per il sistema brasiliano. Esempio di questo è l'articolo 267, VI, che parla che il processo sarà estinto quando mancare una degli condizione della azione.

Così, attualmente, come abbiamo detto, la strutura del sistema processuale civile brasiliano sembra molto la strutura del sistema processuale civile italiano, cosa permètere un studio di diritto comparato molto efficace.

Possiamo dire, allora, che nostro codice ha, in verità, quatre libri, già che il quinto libro c'è solamente dieci articoli che parlano di disposizioni transitorie. Il libro primo parla del processo de conhecimento, dove ci sono disposizione del primo e del secondo libro del codice italiano. Nostro secondo libro parla del processo d' esecuzione, il terzo del processo cautelare e il quarto dei procedimenti speciali.

Nonostante sai un ottimo codice quanto a la tècnica e quanto al contenuto avanzato, tanto che ha dedicato un libro proprio al processo cautelare, diventando legge quello che la dottrina già aveva riconosciuto da molto tempo come una terza spècie di processo, le modificazione nella società hanno esigeruto una adattazione agli nuove idea, fondata nella agilità e efetività del processo.

Allora, nel 1995 sono state editate vari leggi che la dottrina ha chiamato 'la riforma del codice di procedure civile', che hanno alterato istituti antichi e introduto nuovi istituizioni ancora non esistente nel sistema atuali.

Così, il procedimento d'ingiunzione, che como ho detto per noi si chiama "ação monitória", è stato introduto nel codice per la Legge n. 9.079, di 14.07.1995, come si può nuotare nel texto che li ho distributo.

Come istituizione nuova, il suo profilo non è stato ancora giusto nella dottrina brasiliana. $\mathrm{Ci}$ sono molti litigi sull'alcuni punti di verà importanza, già largamenti tratati per la dottrina italiana, specialmente per Calamandrei (1926) i Garbagnati (1979). In verità, le lezioni di 
questi i di altri attori italiani saranno importantissimi, sono sicuro, dopo alcuni adattamenti, per lo svilupo del tema in Brasile.

Partendo di questo panorama, non parlerò sulli problemi come la natura del procedimento, esistenza del titolo ingiuntivo, de la cosa giudicata oppure della natura dela nostra opposizione, dove la dottrina italiana è molto di più avanzata. Farò, soltanto, una comparazione tra la legge brasiliana i la legge italiana eppure di suoi principali differenze.

Così, dobbiamo prendere che la legge brasiliana ha fatto una opzione per la azione monitoria documentale, non accetando la chiamata azione monitoria pura del diritto tedesco. Ossia, per noi soltanto è possibile chiedere la ingiunzione quando l'atore ha prova scritta, come dice l'articolo 1.192a.

Però, la legge brasiliana non ha detto cosa si deve capire sulla locuzione prova escrita, come fa la legge italiana, in parte, negli articoli 634 da 636. Per questo il compito di stabilire la portata della locuzione è stato atribuito alla dottrina e giurisprudenza.

Mi sembra che una degli migliori apporti è stata fatta per il professore e giudice Batista Lopes (1999, página), che há detto:

De todo exposto é lícito concluir que a prova escrita a que se refere a lei processual não é apenas a constituída pelo devedor ou dele emanada. Ao juiz caberá verificar, em cada caso, se os documentos apresentados pelo autor, ainda que produzidos por terceiros, têm o condão de estabelecer vínculo jurídico idôneo a autorizar a expedição, initio litis, de mandado de pagamento.

\section{Ossia:}

Di tutto che se ha detto è licitto concludere che la prova scritta alla quale si riferisci la legge processuale no è soltanto quella che è construita per il debitore opure di lui emanata. Al giudice tocca verificare, in ogni caso, si i documenti presentati per l'atore, ancora che prodotto por terzo, può stabilire rapporto giuridico idoneo che possa autorizzare la pronuncia, 'initio litis', di decreto di pagamento.

Però, di qualsiasi modo, una cosa è giusta. L'articolo 1.102a, quando parla in prova scritta senza efficacia esecutiva, vieta la domanda si il 
documento ancora permete al'attore propore diretamente l'esecuzione, al'oposto di quello che sucede nel diritto italiano, como permite l'articolo $641,3^{\circ}$ Comma, epure 642, $1^{\circ}$ Comma.

La matteria che può essere oggeto della "ação monitória" e la stessa che può essere oggeto del procedimento ingiuntivo, ossia somma di danaro, cose fungibile opure cosa mobile determinata. La redazione del'articolo $1.102 \mathrm{a}$ brasiliano, in questo punto, sembra molto la redazione del'articolo $633,1^{\circ}$ comma, italiano. Ambedue vietano l'ingiunzione quando il bene è imobile.

Però, quando parla di somma in danaro, la legge brasiliana non dicce niente sulla liquidità (liquidezza) come fa la legge italiana. Dunque si ha capito, come ricorda il professore Talamini (1998, p. 80), che se il valore non è liquido, il giudice deve rigettare la domanda.

Niente vieta l'uso della ação monitória per lo Stato e degli enti pubblici, ma grande parte della dottrina dice che la domanda non può essere fatta davanti loro, dunque la "Fazenda Pública" c'è un procedimento proprio per essere esecutada. Ormai ci sono un bruto litigio nella dotttrina su questo punto, che ancorra non è stato accertato di modo finale per gli Tribunali.

Quanto al procedimento, l'atore propone la domanda obbedicendo i requisiti generali della legge brasiliana - requisitos da petição inicial - che ci sono nell'articolo 282, contenendo ancora la prova scritta, come sucede in Italia (articolo 638). Però, non c'è previsione esplicita della necessità di dichiarare la somma di danaro che l'atore è disposto ad accetare in mancanza della prestazione in natura. Ormai è possibile aplicare, analogicamente, l'articolo 627 , che dice che l'atore há diritto al rissarcimento e a eventuale indenisazione.

Allora, com l'accoglimento della domanda il giudice ingiunge il pagamento o consegna nel termine di 15 (quindici) giorni, quando il debitore potra propore l'opposizione, che per noi si chiama "embargos ao mandado", suspendendo la efficacia esecutiva del decreto.

Faccio vedere, qui, che il termine processuale di 15 giorni è giusto davanti il sistema giuridico brasiliano, dove i termine sono di solito minori che nel sistema italiano. Ma nostro giudice no ha il potere di dilatare oppure abbreviare il termini di 40 giorni per giusti motivi, come ha il giudice italiano (articolo $641,2^{\circ}$ comma). 
Anchè non abbiamo la possibilità di fare opposizione tardiva, come dice l'articolo $650,1^{\circ}$ comma, ormai che c'è molto rigore in Brasile com il sistema di contumacia, quindi abbiamo adotato Qui la stessa regolamentazione del sistema austriaco i tedesco.

Per noi (articolo 1.102 c, $\S 2^{\circ}$ ), come in Italia (articolo 645, $2^{\underline{a}}$ comma), il procedimento si svolge secondo le norme del procedimento ordinario, ma purttroppo non c'è la possibilità di redure i termini di comparizione a metà.

Se la domanda che è stata fatta negli "embargos ao mandado", ossia nela nostra oposizione, è procedente, il procedimento di ingiunzione è canccelato. Ma si manca la opposizione opure non è acettata, allora si costitui di pieno diritto il titolo esecutivo giudiciale. L'articolo 1.102c i il suo $\S 3^{3}$ ci sono espresso a questo rispetto. Dunque non c'è necessità di più nessuno atto processuale. Questa è da dottrina più forte, ma quanto più studio il tema più dubbia ho su questa posizione.

Così, finita la prima fase del procedimento, dice l'articolo $1.102 \mathrm{c}$, $\S 3^{2}$, che la fase esecutiva deve seguire quello che determina il "Livro II, Título II, Capítulos II e IV”, che tratano dell'escuzione per consegna di cosa i dell'esecuzione per espropriazione forzata, che sembrano il sistema italiano.

La dottrina più forte sostenta che è possibile propore opposizione all'esecuzione nella fase esecutiva della "ação monitória". Però, credo che la legge brasiliana, por non parlare espressamente nel "Capítulo V", che trata dell'oposizione all'esecuzione, ha vietato questo procedimento.

Così, sperando avere datto una picola ideà dell procedimento d'ingiunzione brasiliano, più una volta ingracio il professore Saletti per l'invito i a tutti per l'atenzione. Firmo Qui. Grazie mille.

\section{CONCLUSÃO}

Embora este texto se preste mais a demonstrar, publicado no Brasil, exatamente o contrário do que se pretendia demonstrar na Itália, ou seja, trazer a público uma pequena noção do procedimento de injunção no direito italiano, cremos que o mesmo objetivo será atingido, colocando os leitores em contato com alguns problemas de um sistema jurídico alienígena, que muitas vezes acabam por se repetir entre nós. 
Da mesma forma, atingimos nosso segundo objetivo, homenageando os professores já citados e, ainda, alimentando em nossa mente e em nosso coração os sentimentos de admiração, saudade e respeito acadêmico que sempre uniram juristas brasileiros e italianos, na busca de soluções eficientes para os problemas do Direito Processual Civil.

\section{REFERÊNCIAS}

BATISTA LOPES, J. A prova escrita na ação monitória. Caderno de doutrina da Tribuna da Magistratura. Jul./ago./1999.

CALAMANDREI, P. Il procedimento monitorio nella legislazione italiana. Milano: Società Editrice 'UNITAS'. 1926.

FREDERICO MARQUES, J. O direito processual civil em São Paulo. São Paulo: Saraiva, 1977.

GARBAGNATI, E. I procedimenti d'ingiunzione e per canvalida di sfratto. Milano: Giufrè, 1979.

TALAMINI, E. Tutela monitória. São Paulo: Revista dos Tribunais, 1998. 


\section{ANEXO - LEGISLAÇÃO ITALIANA CITADA}

627. Riassunzione. - I1 processo esecutivo deve essere riassunto con ricorso nel termine perentorio fissato dal giudice dell'esecuzione [630] $\mathrm{i}$, in ogni caso, non più tardi di sei mesi dal passaggio in giudicato della sentenza di primo grado o dalla comunicazione della sentenza di appello che rigetta l'opposizione [481, 549, 601].

633. Condizioni di ammissibilità. - Su domanda di chi è creditore di una somma liquida di danaro [638] o di una determinata quantità di cose fungibili [639], o di chi ha diritto alla consegna di una cosa mobile determinata, il giudice competente [637] pronuncia ingiunzione di pagamento o di consegna [186ter]:

1) se del diritto fatto valere si dà prova scritta $[634,635]$;

2) se il credito riguarda onorari per prestazioni giudiziali o stragiudiziali o rimborso di spese fatte da avvocati, procuratori, cancellieri, ufficiali giudiziari o da chiunque altro ha prestato la sua opera in occasione di un processo $[636,637]$.

634. Prova scritta. - Sono prove scritte idonee a norma del numero 1) dell' articolo precedente le polizze e promesse unilaterali per scrittura privata [c.c. 1988, 2702] e i telegrammi [c.c. 2705], anche se mancanti dei requisiti prescritti dal Codice civile.

Per i crediti relativi a somministrazioni di merci e di danaro nonché per prestazioni di servizi fatte da imprenditori che esercitano un' attività commerciale [c.c.2195], anche a persone che non esercitano tale attività, sono altresì prove scritte idonee gli estratti autentici delle scritture contabili di cui agli articoli 2214 e seguenti del Codice civile, purché bollate e vidimate nelle forme di legge e regolarmente tenute, nonché gli estratti autentici delle scritture contabili prescritte dalle leggi tributarie, quando siano tenute con l'osservanza delle norme stabilite per tali scrittiure [186ter].

635. Prova scritta per i crediti dello Stato e degli enti pubblici. - Per i crediti delo Stato, o di enti o istituti soggetti a tutela o vigilanza dello Stato, sono prove idonee anche i libri o registri della publica 
amministrazione, quando un funzionario all' uopo autorizaato o un notaio ne attesta la regolare tenuta a norma delle leggi e dei regolamenti. Restano salve le disposizione delle leggi sulla riscossione delle entrate patrimoniali dello Stato e degli enti o istituti sopra indicati.

Per i crediti derivanti da omesso versamento agli enti di previdenza e di assistenza dei contributi relativi ai rapporti indicati nell' articolo 459, sono altresì prove idonee gli accertamenti eseguiti dal' Ispettorato del lavoro e dai funzionari degli enti.

636. Parcella delle spese e prestazioni. - Nei casi previsti nei nn. 2 e 3 dell' articolo 633, la domanda deve essere accompagnata dalla parcella delle espese e prestazioni, munita della sottoscrizione del ricorrente e corredata dal parere della competent associazione professionale. I1 parece non occorre se l' ammontare delle spese e delle prestazioni è determinato in base a tariffe obbligatorie.

I1 giudice, se non rigetta il ricorso a norma dell' articolo 640, deve attenersi al parere nei limiti della somma domandata, salva la correzione degli errori materiali.

638. Forma della domanda e deposito. - La domanda d' ingiunzione si propone con ricorso contenente, oltre i requisiti indicati nell' articolo $125, l^{\prime}$ indicazione delle prove che si producono $[634,635]$. I1 ricorso deve contenere altresì l' indicazione del procuratore del ricorrente oppure, quando è ammessa la costituzione di persona [82, 86], la dichiarazione di residenza o l' elezione di domicilio nel comune dove ha sede il giudice adito.

Se manca l' indicazione del procuratore oppure la dichiarazione di residenza o l' elizione di domicilio, le notificazioni al ricorrente passono essere fatte presso la cancelleria [645; att. 188].

11 ricorso è depositato in cancelleria insieme con i documenti che si allegano; questi non passono essere ritirati fino alla scadenza del termine stabilito nel decreto d' ingiunzione a norma dell' articolo 641.

641. Accoglimento della domanda. - Se esistono le condizioni previste nell' articolo 633, il giudice, con decreto motivato, ingiunge all'altra parte di pagare la somma o di consegnare la cosa o la quantità di cose chieste o invece di queste la somma di cui all' articolo 639 nel termine di quaranta giorni, con l' espresso avvertimento che nello 
stesso termine può essere fatta opposizione a norma degli articoli seguenti e che, in mancanza di opposizione, si procederà a esecuzione forzata [483 ss.].

Quando concorrono giusti motivi, il termine può essere ridotto sino a dieci giorni oppure aumentato a sessenta. [Se l' intimato risiede nelle province libiche $o$ in territori soggeti alla sovranità italiana, il termine non può essere minore di trenta, né maggiore di centoventi giorni].

Nel decreto, [eccetto per quello emesso sulla base di titoli che hanno già efficacia esecutiva secondo le vigenti disposizioni], il giudice liquida le spese e le competenze e ne ingiunge il pagamento.

642. Esecuzione provvisoria. - Se il credito è fondato su cambiale, assegno bancario, assegno circolare, certificato di liquidazione di borsa, o su atto ricevuto da notaio o da altro pubblico ufficiale autorizzato, il giudice, su istanza del ricorrente, ingiunge al debitore di pagare o consegnare senza dilazione, autorizzando in mancanza l' esecuzione provvisoria del decreto e fissando il termine ai solo effetti dell' opposizione [655].

645. Opposizione. - L'opposizione si propone davanti all'uffico giudiziario al quale appartiene il giudice che ha emesso il decreto, con atto di citazione notificato al ricorrente nei luoghi di cui all' articolo 638. Contemporaneamente l' ufficiale giudiziario deve notificare avviso dell' opposizione al cancelliere affinché ne prenda nota sull' originale del decreto.

In seguito all' opposizione il giudizio si svolge secondo le norme del procedimento ordinario davanti al giudice adito; ma i termini di comparizione sono ridotti a metà $[163$ bis, $165,166,313]$.

650. Opposizione tardiva. - L'intimato può fare opposizione anche dopo scaduto il termine fissato nel decreto [641], se prova di non averne avuta tempestiva conoscenza per irregolarità della notificazione o per caso fortuito o forza maggiore. 
